\title{
ON A NOTE OF MARCUS CONCERNING A PROBLEM POSED BY FRINK
}

\author{
RICHARD B. DARST AND ROBERT E. ZINK ${ }^{1}$
}

In his measure-theoretic treatment of Riemann integration, Frink has shown that the set of discontinuities of a Riemann integrable function is a countable union of sets of zero Jordan content [2]. Such a set is thus of Lebesgue measure zero and of the first category. By constructing a counterexample in the plane, Frink also established the fact that not every first category null set is the set of discontinuities of some Riemann integrable function. In the course of his consideration of Jordan content and Riemann integration in topological measure spaces, Marcus has proved the direct generalization of the positive assertion above [5] and has addressed himself to the converse proposition in this more general setting [6]. In the latter investigation, restricting his attention to somewhat special topological measure spaces, Marcus was able to establish the following proposition:

THEOREM 1. Let $X$ be a complete, separable metric space, and let $\mu$ be a complete $\sigma$-finite measure defined on the ( $\mu$-completion of the) Borel $\sigma$-algebra of subsets of $X$ that assigns measure 0 to each finite set. If, in addition, (*) $X$ contains a perfect nowhere dense set $P$ such that $\mu(S \cap P)$ $>0$, whenever $S$ is an open ball that meets $P$ nonvacuously, then $X$ contains a first category null set $B$ that cannot be written as a denumerable union

$$
B=\bigcup_{n=1}^{\infty} B_{n}
$$

with $\mu\left(\operatorname{Fr} B_{n}\right)=0$ for all $n$.

The set $B$, which is called a set of Frink type, presented in the proof of this theorem is a $G_{\delta}$. After pointing out the obvious impossibility of finding a Frink set of type $F_{\sigma}$, Marcus asked whether there exist Frink sets of various Borel types, whether there exist non-Borel sets of Frink type and whether there exist nonanalytic Frink sets. He also expressed an interest in the potency of the set $\mathcal{F}$ of all sets of Frink type and suggested that the problem be investigated in the nonseparable case. In the sequel we are able to answer affirma-

Received by the editors July 14, 1964.

1 The authors are supported by the National Science Foundation (NSF GP-1665). 
tively each of the first three questions, under the general hypotheses set down by Marcus, and we find without difficulty that the cardinal number of $\mathfrak{F}$ is $2^{c}$. Moreover, we are able to strengthen Theorem 1 by proving that the condition $\left({ }^{*}\right)$ is implied by the other hypotheses of that theorem. The article concludes with a demonstration of the existence of sets of Frink type in certain nonseparable metric spaces.

The discussion that follows is a considerably simplified version of the original work. The authors wish to acknowledge their indebtedness to the referee for the helpful suggestions that resulted in this simplification.

Theorem 2. Let $X$ be a complete, separable metric space, and let $\mu$ be the completion of a Borel measure in $X$ that vanishes for finite sets. Suppose further that $0<\mu(E)<\infty$ for some measurable set $E$. Then $X$ contains Frink sets of every Borel type $G_{\alpha}$ with $\alpha \geqq 1$ (as well as non-Borel analytic, and nonanalytic Frink sets).

Proof. By $[3,183] E$ contains a compact set $C$ of positive finite measure. By $[1,84]$ there exist $M$, a null subset of $C$, and a homeomorphism $\phi$ of $C-M$ onto a subset of the interval $[0, \mu(C)]$ such that $\mu$ corresponds to Lebesgue measure, $m$, in $\phi(C-M)$. Then $\phi(K)$ is a compact linear set of positive measure. It is well known that in $\phi(K)$ we can construct two disjoint perfect sets $C_{1}$ and $C_{2}$, both nowhere dense relative to $\phi(K)$, such that $C_{2}$ has zero measure and such that no open interval meets $C_{1}$ in a nonempty null set. Let $A$ be a $G_{\delta}$ null set that is dense in $C_{1}$ and let $B$ be an arbitrary subset of $C_{2}$. Then $F=A \cup B$ is a null set of the first category in $\phi(K), A$ is a Frink set [6] and the closure of $B$ is a null set. Consequently, $F$ is a Frink set relative to $\phi(K)$, and it follows that $\phi^{-1}(F)$ is a Frink set relative to $X$. Since $B$ can be any subset of $C_{2}$, the set $\phi^{-1}(F)$ may belong to any of the classes mentioned in the statement of the theorem (see, for example, $[4,20]$ and $[8,230])$. Moreover, the cardinality of the class of all Frink sets in $X$ is seen to be $2^{c}$. Finally, the set $\phi^{-1}\left(C_{1}\right)$ satisfies $(*)$.

The referee has observed that any separable metric space $X$ admitting a nontrivial Borel measure $\mu$ that vanishes for finite sets, contains a perfect nowhere dense set $P$ such that $\mu(S \cap P)>0$ whenever $S$ is an open ball that meets $P$ nonvacuously. It suffices to embed $X$ as a dense subset of a complete metric space $Y$ and to define a Borel measure $\nu$ in $Y$ by setting $\nu(E)=\mu(E \cap X)$. Since $Y$ contains a perfect nowhere dense set $P$ with the stated property, it follows that the set $P$. $X$ has the same property relative to $X$.

In [6], Marczewski and Sikorski have defined the separability 
character of a topological space to be the smallest element of the set of all cardinal numbers each of which is the potency of some basis for the open sets. We remark that the work of the above cited authors suffices to show that the restriction on the separability character of $X$ (in Theorem 1) can be weakened to an exceedingly mild condition.

THEOREM 3. Let $X$ be a complete metric space the separability character of which is a nonmeasurable cardinal. If $\mu$ is a nontrivial $\sigma$-finite measure defined on $\Sigma, a \sigma$-algebra of subsets of $X$, if $\Sigma$ contains the class of all open sets and if $\mu$ vanishes on each finite set, then $X$ contains sets of Frink type.

Proof. Under these conditions, Marczewski and Sikorski have proved that $X$ contains a closed separable subspace of positive measure [7]. Thus, working within this subspace, we have only to apply the argument of Marcus in order to obtain the desideratum.

\section{REFERENCES}

1. N. Bourbaki, Éléments de mathêmatique, Livre VI, Chapitre 5, Hermann, Paris, 1956.

2. O. Frink, Jr., Jordan measure and Riemann integration, Ann. of Math. 34 (1933), 518-526.

3. P. R. Halmos, Measure theory, Van Nostrand, New York, 1950.

4. F. Hausdorf, Set theory, Chelsea, New York, 1957.

5. S. Marcus, La mesure de Jordan et l'intégrale de Riemann dans un espace mesure topologique, Act a Sci. Math. 20 (1959), 156-163.

6. - Asupra unei probleme puse de O. Frink Jr., Com. Acad. R. P. Romine 12 (1962), 281-286.

7. E. Marczewski and R. Sikorski, Measures in non-separable metric spaces, Colloq. Math. 1 (1948), 133-139.

8. W. Sierpinski, General topology, Univ. of Toronto Press, Toronto, 1956.

Purdue University 